\title{
Fine Structure of Core Loss Excitations in EELS by Monte Carlo Simulation
}

\author{
M. Attarian Shandiz ${ }^{1}$, F. Salvat ${ }^{2}$ and R. Gauvin ${ }^{1}$ \\ ${ }^{1}$ Department of Materials Engineering, McGill University, Montreal, Canada. \\ ${ }^{2}$ Facultat de Fisica (ECM), Universitat de Barcelona, Barcelona, Spain.
}

Electron energy loss spectroscopy (EELS) gives useful information about the electronic structure and composition of materials. The combination of optical data models for describing electron energy losses with Monte Carlo (MC) simulation provides a versatile method to improve the analysis of EELS spectra [1]. The optical oscillator strength (OOS) is the main input for the optical data models [1]. In this study OOSs were obtained by combining optical properties calculated from density functional theory (DFT) and X-ray photoelectric data. Figure 1a shows the calculated energy loss function of NiO performed by WIEN2k code [2]. NiO is a strongly correlated system; hence in the DFT calculation we used the new modified Becke-Johnson (mBJ) exchange potential [3] and the local density approximation (LDA) correlation potential [2]. In comparison with the generalized gradient approximation (GGA), the new exchange potential gives results in better agreement with experimental data. Figure 1b shows the calculated OOS obtained by a combination of data from our DFT calculation and X-ray photoelectric data [4], and applying Bethe sum rule.

Fine structure of core loss excitation in EELS gives valuable information about atomic bonds and their electronic structure. Using X-ray photoelectric data cannot provide enough resolution to study the effect of parameters of experimental EELS such as thickness and collection semi-angle on the fine structure of core loss excitations in MC simulations. Hence, the fine structure was included in the MC simulated EELS spectra through a new approach. The OOS was refined using the experimental EELS spectra. After background removal from experimental data [5] and applying a single scattering approach for thin specimen the OOS with fine structure was obtained. The obtained OOS can be normalized by comparison to the area under the edges from the OOS obtained from X-ray photoelectric data and thereafter it can be replaced in the original OOS presented in Figure 1b. The same approach was used for the simulated shape of edges obtained from DFT calculations; however in this case is not necessary to use the single scattering approach. As another approach, the height of added experimental edges was fixed equal to the height of edges obtained from the X-ray photoelectric data. The LEEPS code [6] was adapted to perform MC simulation of EELS spectra of NiO for different thicknesses. Generally, all these three methods predict the effect of thickness on the shape of core loss edges well by introduction of background signal as observed in Figure 2. Thus with the proposed approaches the fine structure of edges can be simulated through the refined OOS and using it for $\mathrm{MC}$ simulations.

\section{References}

[1] M. Attarian Shandiz, Microsc. Microanal. 18 (2012), p. 998-999.

[2] P. Blaha et al, WIEN2k, University of Technology Vienna, Austria, 2001.

[3] W. Hetaba, et al, Phys. Rev. B 85 (2012), p. 205108.

[4] C.T. Chantler et al, NIST Standard Reference Database 66, 2005.

[5] D. Huber et al, "Relative Sensitivity of XEDS vs EELS in the AEM ", M\&M 2010, Portland.

[6] J. M. Fernández-Varea et al, Nucl. Instrum. and Meth. B 108 (1996), p. 35-50.

[7] F. Parmigiani et al, Solid State Communications 96 (1995), p. 161-165. 

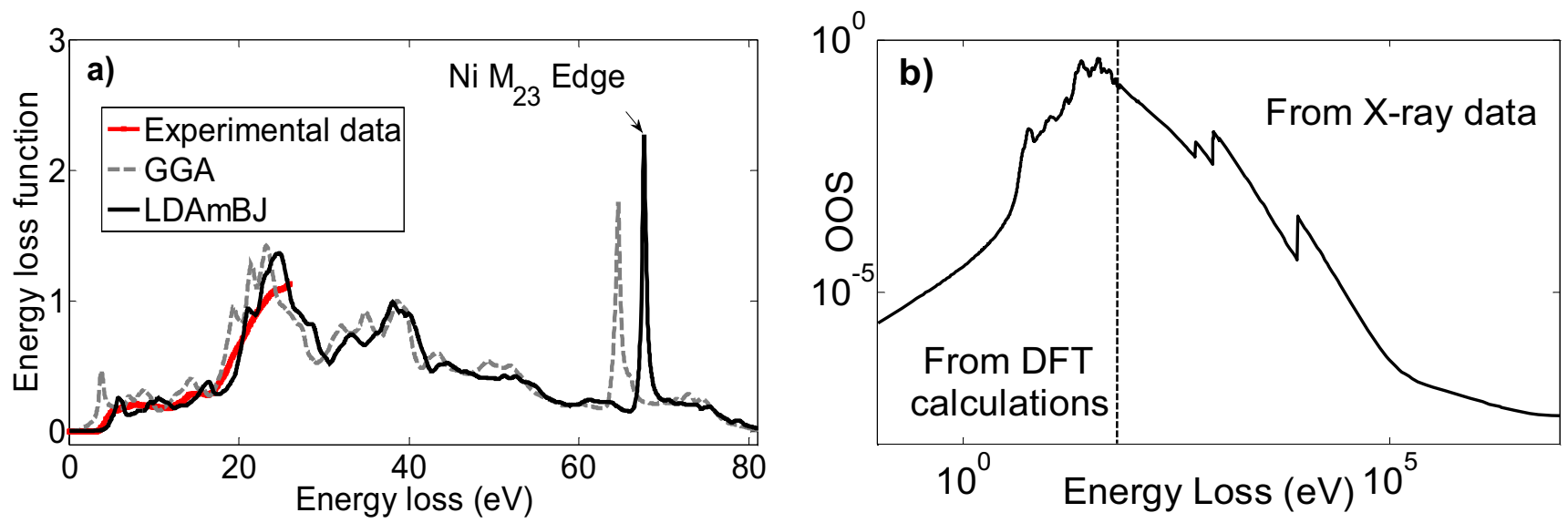

FIG.1. a) Comparison of the energy loss function, of $\mathrm{NiO}$ calculated using GGA and LDA with mBJ exchange-correlation potential with experimental data [7]. b) OOS of $\mathrm{NiO}$ calculated by combination of optical data calculated from DFT calculations and X-ray photoelectric cross section [4]. The dashed line indicates the $65 \mathrm{eV}$.
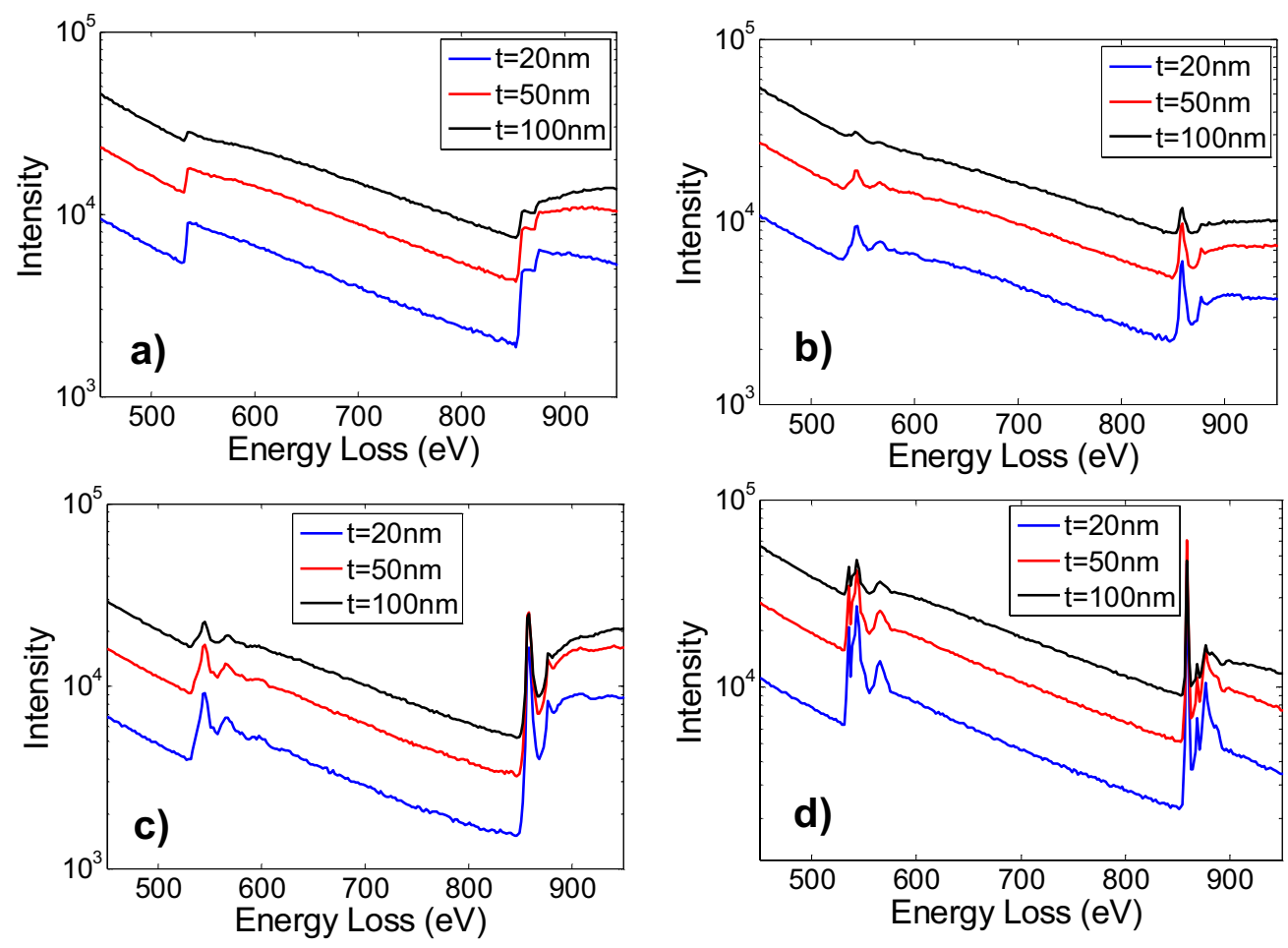

FIG. 2. Fine structure $\mathrm{MC}$ simulations of $\mathrm{O} \mathrm{K}$ and $\mathrm{Ni} \mathrm{L}_{2-3}$ edges using four methods: a) X-ray photoelectric data $b$ ) fixed height experimental data c) normalized experimental data d) normalized DFT simulation data for different thicknesses $(\mathrm{t})$. 